\title{
Molecular tools and genetic markers for the generation of transgenic sexing strains in Anopheline mosquitoes
}

Federica Bernardini, Roya Elaine Haghighat-Khah, Roberto Galizi, Andrew Marc Hammond, Tony Nolan and Andrea Crisanti*

\begin{abstract}
Malaria is a serious global health burden, affecting more than 200 million people each year in over 90 countries, predominantly in Africa, Asia and the Americas. Since the year 2000, a concerted effort to combat malaria has reduced its incidence by more than $40 \%$, primarily due to the use of insecticide-treated bednets, indoor residual spraying and artemisinin-based combination drug therapies. Nevertheless, the cost of control is expected to nearly triple over the next decade and the current downward trend in disease transmission is threatened by the rise of resistance to drugs and insecticides. Novel strategies that are sustainable and cost-effective are needed to help usher in an era of malaria elimination. The most effective strategies thus far have focussed on control of the mosquito vector. The sterile insect technique (SIT) is a potentially powerful strategy that aims to suppress mosquito populations through the unproductive mating of wild female mosquitoes with sterile males that are released en masse. The technique and its derivatives are currently not appropriate for malaria control because it is difficult to sterilise males without compromising their ability to mate, and because anopheline males cannot be easily separated from females, which if released, could contribute to disease transmission. Advances in genome sequencing technologies and the development of transgenic techniques provide the tools necessary to produce mosquito sexing strains, which promise to improve current malaria-control programs and pave the way for new ones. In this review, the progress made in the development of transgenic sexing strains for the control of Anopheles gambiae, a major vector of human malaria, is discussed.
\end{abstract}

Keywords: Malaria, Anopheles mosquitoes, sex determination, genetic engineering, vector control

\section{An evolutionary perspective of sex and sex determination in insects}

Reproduction is a fundamental feature for life to subsist. In the majority of eukaryotes, sexual reproduction has evolved to allow, through meiosis and fusion of gametes, the rapid appearance of new genetic traits [1]. Sex-specific life histories and adaptations can result in highly specialised sexes that act to maintain this reproduction strategy [2-4].

A common feature of sex determination is a signalling cascade that begins with a singular primary signal and results in the expression of several hundred sexually dimorphic traits, often via a set of sex-specifically spliced

\footnotetext{
* Correspondence: a.drcrisanti@imperial.ac.uk

Department of Life Sciences, Imperial College London, South Kensington Campus, London SW7 2AZ, UK
}

intermediates. In the model organism Drosophila melanogaster, sexual development is traditionally thought to be initiated by the balance of female determinants on the $\mathrm{X}$ chromosome and male determinants on the autosomes. A more recent work supports the alternative view that the primary sex determining signal in flies is represented by the number of $\mathrm{X}$ chromosomes in the embryo rather than the X:A ratio [5]. Around 2 hours after fertilisation, the primary signal sets the state of activity of the sex lethal $(s x l)$ gene. Sxl is itself a splice factor that acts upon the transformer (tra) transcript to produce a female-specific form of the mRNA that encodes a functional Tra protein. In turn, Tra triggers splicing of doublesex $(d s x)$ pre-mRNA resulting in expression of the female-specific isoform, $\mathrm{Dsx}^{\mathrm{F}}$ protein. In its capacity as a transcription factor, $\mathrm{Dsx}^{\mathrm{F}}$ leads to the 
development of females. In the absence of Sxl protein, the primary transformer transcript is spliced to produce an mRNA that does not encode functional Tra protein. This consequently determines splicing of the $d s x$ primary transcript to produce a $\mathrm{Dsx}^{\mathrm{M}}$ transcription factor that enable masculine features [6]. In other organisms, sex determination is due to a trans-acting male-determining gene located on the male sex chromosome, generally identified as the Y. When this gene is expressed, during early embryogenesis, it inhibits factors that destine the sex determination pathway to the default female form, thus determining male development [7]. In the organisms that fall into this category, unlike the situation in Drosophila, the presence of the male chromosome is a crucial factor for determining sex.

Anopheles gambiae is a member of the Anopheles gambiae complex that includes the principal vectors of human malaria [8]. Due to the burden imposed by these mosquitoes, efforts have been made to characterise their biology and special attention has been given to sex determination. In 1979 Baker et al., found that triploid Anopheles culifacies individuals with $3 \mathrm{X}$ chromosomes were phenotypically females whereas XXY individuals showed a male phenotype [9], thus indicating that in mosquitoes the primary signal is different from that which is observed in the Drosophila model. In addition, a number of experiments in several Anopheles species used male inheritance of translocations as evidence pointing to the involvement of the $\mathrm{Y}$ chromosome in sex determination [10-13]. Recently, Krzywinska et al., isolated and characterised a gene, $Y o b$, which acts as a male determination factor in An. gambiae [14]. This gene is located on the $\mathrm{Y}$ chromosome and controls male-specific splicing of $d s x$, the only downstream member of the sex determination cascade known in anopheline mosquitoes [15]. In the Asian malaria vector An. stephensi, a small protein named GUY1 acts as a primary signal that affects embryonic development in a sex-specific manner. This protein is encoded by a Y-linked gene, Guy1, that represents the best candidate for the male-determining factor [16]. In Aedes aegypti, Nix, a dominant male-determining factor located within a Y chromosome-like region called $\mathrm{M}$ locus, has been recently identified [17].

\section{Transgenic sexing strains for vector control}

Vector control is a crucial component of many disease control programmes. Sterile Insect Technique (SIT) is a species-specific and environmentally non-polluting method of insect control which aims to reduce the ability of the targeted species to produce viable offspring [18]. This technology requires repeated releases of mass reared male insects that are generally sterilised using irradiation. By competing with wild males and mating with females over time, sterilised males lead to reduction of the targeted insect population. SIT programs have been successfully used for the control or local elimination of some insect pests such as the tsetse fly [19], medfly [20], melon fly [21] and the screwworm [22].

A major drawback for the application of this technique to mosquitoes is the reduced reproductive competitiveness of radiation-sterilised strains [23, 24]. This contributed to the failure of SIT programs against Anopheles quadrimaculatus in Florida [25, 26] and Culex tritaeniorhynchus in Pakistan [27]. In El Salvador, chemosterization was used as an alternative to induce sterility in Anopheles albimanus with promising results [28], though the toxicity of the chemical's residue was not acceptable for large-scale use. Improvements in mass rearing techniques and irradiation sterilisation, coupled with thorough investigations of important issues, such as gamma ray dosage and male fitness parameters, led to the successful demonstration of population suppression of Aedes albopictus in northern Italy [29]. However, the technique and its derivatives remain inappropriate for malaria control, due to the difficulty to sterilise males without compromising their ability to mate and the lack of a reliable method for mosquito sex separation. The latter is crucial, as the accidental release of female mosquitoes would contribute to disease transmission.

For other technologies, such as Incompatible Insect Technique (IIT), female-free releases are also fundamental [30, 31]. IIT strategies are shown to be valuable for the control of various mosquito species [32,33]. In these IIT methods, sterility is induced by Wolbachia, which is an obligate intracellular bacterium. When infected male mosquitoes mate with uninfected females or with females infected with incompatible Wolbachia strains, the microorganism induces cytoplasmic incompatibility, a phenomenon that results in embryonic lethality. Accidental releases of female mosquitoes infected with the Wolbachia strain used for the sterilisation program would interfere with the effectiveness of IIT and could potentially cause its failure.

Irradiation and classical genetics have been exploited to generate efficient genetic sexing strains in a number of tephritid species [21,34-37]. A genetic sexing strain for Anopheles arabiensis mosquitoes has been established based on dieldrin resistance; however, there are some concerns over the use of this strain for large scale application that revolves around the use of the insecticide [38]. Other methods based on mechanical, behavioural and developmental approaches have been developed, however, the natural variation of parameters used for sex separation, particularly high for some mosquito species, poses a limit to their relatability [39]. The generation of transgenic sexing strains where inherited traits, such as fluorescent markers or resistance to drugs, are specifically associated with one of the two sexes 
could be used as a high throughput method for mosquito sex separation and facilitate the releases of male-only mosquitoes for SIT based approaches.

An alternative strategy based on the knowledge of the molecular mechanisms involved in the sex determination pathway, has been successfully exploited to develop a transgene-based female-specific lethality system for early embryonic sexing in medfly. The system relies on the sex-specifically spliced transformer intron to restrict the expression of a lethal gene only in females [40]. In An. gambiae, injections of the male-determining factor, $Y o b$, into young embryos are lethal to females [14]. Similarly, in An. stephensi, ectopic expression of Guy1 confers 100\% female lethality during embryonic and early larvae stages [41]. Ectopic expression of Nix in Ae. aegypti gives masculinized females [17]. This female-killing property could be used to eliminate harmful female mosquitoes or convert them into harmless males.

A similar principle led to the development of the sex-ratio distortion technique in mosquitoes. In 2014, Galizi et al. generated a transgenic An. gambiae strain expressing the endonuclease IPpo-I under the testes-specific $\beta 2$-tubulin promoter. IPpo-I recognises and cuts ribosomal rDNA repeats located in a single cluster on the An. gambiae X chromosome. Upon IPpo-I activity, only $\mathrm{Y}$ chromosome-bearing sperm remain functional, enabling transgenic males to generate only male progeny [42]. A similar result was achieved using a CRISPR-Cas9 sex distortion system [43]. If the sex-ratio distorter is linked to the $\mathrm{Y}$ chromosome, this technique has the potential to quickly spread and suppress a wild population even if a very low number of male mosquitoes are released [44, 45]. Unfortunately, the biology and complex structure of the Anopheles Y chromosome has hampered progress in this field and restricted the expression of the sex-ratio distorter from an autosomal location.

Mosquito sexing strains are urgently needed for the improvement of existing malaria control strategies and for the development of new ones [46]. In the following paragraphs progress toward the generation of sexing strains using transgenic methods is reviewed.

\section{Genetic engineering for the generation of transgenic sexing strains \\ Transposase induced integration}

Nearly 70 years ago, McClintock identified Transposable Elements (TE), which are DNA sequences that are able to change their position within the genome [47]. TEs can be manipulated to allow the insertion of foreign DNA into a genome and their use has been implemented in a variety of research fields [48-53]. The first successful germline transformation system in insects was based on the $P$-element transposon naturally present in the D. melanogaster genome [54]. Subsequently, TEs such as piggyBac (PB) and Tc1/mariner transposons have been characterised in both prokaryotes and eukaryotes where they are typically present in large numbers [55]. Although the first Anopheles transgenic strain was generated using a transposon belonging to the Tc1/mariner family [56], the most widely used system for germline transformation in this organism is PB-based transposition $[57,58]$.

DNA susceptibility to transgene integration varies throughout the genome. This is particularly true when it comes to the $\mathrm{Y}$ chromosome. The An. gambaie $\mathrm{Y}$ chromosome comprises $10 \%$ of the genome and is estimated to be around 26 megabases in size. The accumulation of repetitive sequences, many of which are also present on other chromosomes, has made the assembly of $\mathrm{Y}$ chromosome sequences difficult. To date, only 5 genes have been identified on the $\mathrm{Y}$ chromosome that is mainly constituted by satellite DNAs and transposons [59]. Given its small size and chromatin structure, it is not surprising that $\mathrm{PB}$ integrations onto the Anopheles $\mathrm{Y}$ chromosomes are rarely reported. It has been speculated that because of its heterochromatic nature, the $\mathrm{Y}$ chromosome could be refractory to the random integration catalysed by transposases. In addition, the regulatory regions commonly used to express marker genes to identify transformation events could be subjected to transcriptional silencing when they land on the $\mathrm{Y}$ chromosome. Nevertheless, in 2014, Bernardini et al. characterised an An. gambiae strain carrying a transgene on the $\mathrm{Y}$ chromosome. The transgenic strain was generated through microinjections of wild-type An. gambiae embryos with a plasmid containing $\mathrm{PB}$ inverted repeats flanking the 3xP3-GFP and Actin5C-RFP fluorescent markers. The neuronal promoter 3xP3 leads to strong expression of the GFP from $1^{\text {st }}$ instar larval stage, while Actin5C promoter displays patchy RFP visible mainly in the periphery of the larval gut. In addition, the transgene contains an I-SceI endonuclease recognition site. As a result, only the transgenic male mosquitoes expressed the fluorescence pattern while females were negative for fluorescence [60].

As emphasised above, reliable systems for sex separation of mosquitoes is of paramount importance. Manual and automated sex separation systems based on transgenic male mosquitoes have been previously reported for An. stephensi [61]. This method relies on the presence of fluorescent markers driven by the male specific $\beta 2$-tubulin promoter. Under this condition, fluorescent markers are expressed in the gonads around $3^{\text {rd }}$ instar larval stage. The strong expression of Y-linked fluorescent markers in early stages reduce larval mortality or fitness impairment due to sorting procedures and facilitate sex separation methods. Furthermore, early 
selection minimises the overall number of larvae reared in laboratory conditions thus reducing operational costs. To explore this opportunity, the transgenic strain carrying Y-linked fluorescent markers was used to separate An. gambiae males and females using a Complex Object Parametric Analyser and Sorter (COPAS). Multiple experiments, where over 16,000 larvae were processed for several generations, led to the accurate separation of fluorescent positive males from fluorescent negative females demonstrating the utility of the Y-transgenic strain for high-throughput mosquito sex separation [62].

Marois et al. also showed that a large number of non-transgenic $A n$. gambiae males can be isolated by COPAS if the fluorescent marker is linked to the $\mathrm{X}$-chromosome. In this case, hemizygous males, carrying the X-linked transgene were crossed to wild type females and their progeny sorted into transgenic females and non-transgenic males [63].

A different embryo injection experimental method led to the generation of an independent An. gambiae Y-linked transgenic strain (Galizi et al., unpublished). Once again, $Y$ integration of the transgene was identified by observation of male-only inheritance of the fluorescent markers. To date, the Y strains described here represent the only examples of An. gambiae mosquitoes carrying transgenes on the $\mathrm{Y}$ chromosome. However, the increased knowledge of the $\mathrm{Y}$ chromosome content together with the fast development of molecular tools for genetic engineering might help the generation of transgenic male sexing strains.

\section{$\varphi$ C31-att induced site specific integration}

As previously described, the Y-transgenic strain characterised by Bernardini et al. carries an I-SceI recognition site [60]. The exclusive presence of this site in the transgenic construct facilitated the genetic engineering of the Y chromosome. Targeted knock-in by homology-directed repair was used to modify the transgene and insert an attP recombination signal onto the $\mathrm{Y}$ chromosome. The attP is recognised by the phage $\phi C 31$ integrase and allows site-specific integration of DNA constructs carrying a corresponding attB sequence [64, 65]. This transgenic line offers an alternative to the use of fluorescent markers for high throughput mosquito sex separation. In fact, selectable markers could be specifically inserted onto the $\mathrm{Y}$ chromosome thus conferring different features to males and females. For example, SIT programs for the control of the Mediterranean fruit fly Ceratitis capitata make use of genetic sexing strains containing a Y-linked translocation that rescues a temperature sensitive lethal mutation only in males [66]. The An. gambiae Y-linked attP strain allows for the insertion of such a gene directly onto the $\mathrm{Y}$ chromosome overcoming fitness reduction due to mutagens used to induce translocations. Alternatively, to achieve sex separation in rearing conditions, Y-linked genes conferring resistance to an insecticide could be used $[10,38,67-70]$.

As a number of vector control strategies aim to deliver genes of interest specifically onto the $Y$ chromosome, the Y-attP line represents a promising tool for malaria vector control. For example, site-specific integration onto the $\mathrm{Y}$ chromosome would benefit the efficiency of techniques such as sex distortion [42]. In fact, a sex-distortion system targeting the $\mathrm{X}$ chromosome would be inherited by all the male progeny when integrated onto the $\mathrm{Y}$ chromosome, allowing the transgene to quickly spread into the mosquito population $[44,45]$.

\section{CRISPR-Cas induced homology-directed insertion}

Since 2012, the Clustered Regularly Interspaced Short Palindromic Repeats (CRISPR)/ CRISPR associated gene (Cas) system (CRISPR/Cas or CRISPR) has simplified the process of highly-specific genome engineering [71]. In its simplest form, a single endonuclease called Cas9 is directed to its genomic target by a single 'guide' RNA molecule that has approximately 20 base-pair complementarity to its DNA target. Recently, the technology has been adapted for site-specific knock-in An. stephensi [72] and An. gambiae [73]. Recent evidence suggests that the strategy may be far more efficient than recombinasemediated integration. In a proof-of-principle experiment (Haghighat-Khah, R.E. et al., unpublished), CRISPR-Cas induced knock-in was successfully used to modify the $A n$. gambiae Y chromosome (Fig. 1). The CRISPR guide RNA (gRNA) was designed to target the attP sequence present in the Y-attP transgenic line described above. Further, despite a small sample size, results suggested that the CRISPR-cas9 system was more efficient than site specific integration $\phi C 31$ mediated, producing more transgenic G0 pooled cages than insects injected using $\phi$ C31 (Table 1). This is the first reported use of CRIPR-cas9 system to modify the $Y$ chromosome of any organism. The CRISPRcas9 system provides a method to edit almost any chosen DNA sequence without requiring complex protein engineering and selection procedures. Therefore, this precise and efficient transformation technology can be used to insert sequences into any Y-linked loci such as those recently identified by Hall et al. [59]. This has widespread implications for further developments of any male-biased transgenic system, which could be inserted into any transcriptionally active region on the $\mathrm{Y}$ by redesigning the gRNA target site. The system could also be used to modify the An. gambiae Y-attP line to include carefully positioned LoxP or FRT sites to enable iRMCE (integrase-Recombinase mediated cassette exchange) [74]. This would expand the molecular toolbox for modifying the Anopheles $\mathrm{Y}$ and allow for the direct comparison and characterisations of 

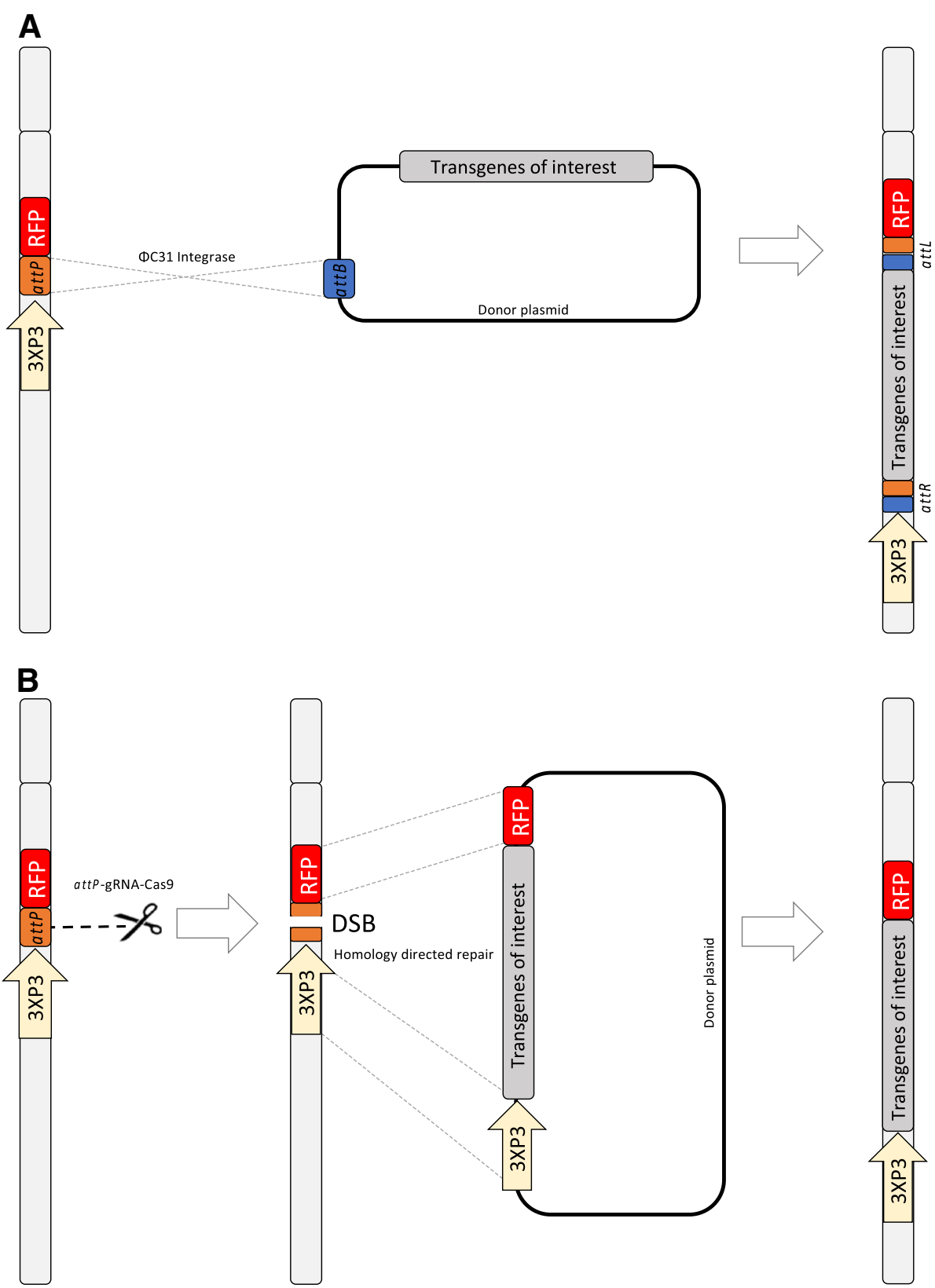

Fig. 1 Schematic view of site-specific integration of transgenes into the $Y$ chromosome of the Anopheles gambiae $Y$-attP strain. The $Y$-attP line carries the attP docking site and a 3XP3-RFP fluorescence marker transcription unit [60]. a The ФC31 integrase, provided in the form of plasmid, catalyses the recombination reaction between the attP target sequence (orange) and attB donor sequence (blue) resulting in the site-specific integration of the transgenic construct onto the $Y$ chromosome. After recombination, hybrid sites called attL and attR are generated, which are no longer recognised by the integrase thus conferring stability to the integration. b A Cas9 coupled with a gRNA (shown as the molecular scissors) induces a double-strand break (DSB) at the attP target site. A donor plasmid containing homologous regions upstream and downstream of the DSB site acts as a template for homology-directed repair. This results in the insertion of the transgenes of interest into the $Y$ chromosome (Table 1; Haghighat-Khah RE et al., unpublished)

components such as lethal, suppressor or refractory effector elements to fine-tune expressed phenotypes.

\section{Transgenic sexing strains for genetic introgression}

The possibility to specifically label sex chromosomes assumes importance for experiments based on classical genetics. In this context, it is often important to track the identity of chromosomes through genetic crosses for successive generations. Given the lack or reduced recombination of sex chromosomes in An. gambaie [75-78], transgenic sexing strains can be used for genetic introgression across sibling mosquito species. 
Table 1 Comparison of integrase-based and nuclease-based approaches to engineer the Y-chromosome of Anopheles gambiae

\begin{tabular}{|c|c|c|c|c|c|c|c|}
\hline Donor plasmid & $\begin{array}{l}\text { Helper } \\
\text { Plasmid }\end{array}$ & $\begin{array}{l}\text { Injected G0 } \\
\text { embryos }\end{array}$ & $\begin{array}{l}\text { Surviving G0 } \\
\text { larvae }\end{array}$ & $\begin{array}{l}\text { Transient G0 } \\
\text { Larvae }\end{array}$ & $\begin{array}{l}\text { Transient G0 } \\
\text { adult } \hat{\partial}\end{array}$ & Pooled cages & Transgenic G1s \\
\hline \multicolumn{8}{|l|}{ A } \\
\hline \multirow[t]{2}{*}{33 nM pHomeT } & $35 \mathrm{nM}$ & 1769 & 134 & 44 & 29 & 5 & $16 / 6559$ \\
\hline & $\varphi C 31$ integrase & & & & & & $\begin{array}{l}1 \text { pooled G0 } \\
\text { transient cage }\end{array}$ \\
\hline \multirow[t]{2}{*}{$33 \mathrm{nM}$ pHomeT } & 35 nM & 600 & 127 & 68 & 29 & 6 & $63 / 5147$ \\
\hline & $\operatorname{attP-gRNA}$ & & & & & & $\begin{array}{l}3 \text { pooled G0 } \\
\text { transient cages }\end{array}$ \\
\hline \multicolumn{8}{|l|}{ B } \\
\hline \multirow[t]{2}{*}{40 nM attBCFP-VasaGFP } & $96 \mathrm{nM}$ & $\sim 2000$ & $\mathrm{n} / \mathrm{a}$ & $\mathrm{n} / \mathrm{a}$ & 5 & $\mathrm{n} / \mathrm{a}$ & $6 / 791$ \\
\hline & QC31 integrase & & & & & & \\
\hline \multirow[t]{2}{*}{$52 \mathrm{nM} 3 \times \mathrm{xP} 3[$ AttP]RFP } & $90 \mathrm{nM}$ & $\sim 4000$ & $\mathrm{n} / \mathrm{a}$ & $\mathrm{n} / \mathrm{a}$ & 15 & $\mathrm{n} / \mathrm{a}$ & $11 / 6160$ \\
\hline & I-Scel & & & & & & \\
\hline
\end{tabular}

(A) ФC31-att recombinase was used to insert a vector, pHomeT, containing the attB donor sequence into the $\mathrm{Y}$-attP strain, carrying the complementary attP target sequence on the Y-chromosome. CRISPR-cas9-directed knock-in was used to integrate the pHomeT plasmid into the same $\mathrm{Y}$ locus. (B) $\Phi C 31$-att recombinase was used to integrate a vector, attBCFPVasaGFP, containing the attB donor sequence into the $Y$-attP strain. I-Scel-directed knock-in was used to integrate the vector 3xP3[AttP]RFP into the Y chromosome of T4 strain, carrying the I-Scel recognition site [60].

Anopheles arabiensis is another important vector of malaria. Rearing this species in lab settings is widely known to be challenging and genome sequences have not yet been assigned to chromosomes [79, 80]. Despite the medical importance of this species, there has been little progress in the application of molecular tools for its DNA modification, and to date no transgenic strain is available. Recently, Bernardini et al described a number of genetic crosses that allowed the introgression of the An. gambiae Y chromosome into the An. arabiensis genetic background [62]. For this purpose, the previously described $A n$. gambiae Y-transgenic strains carrying Y-linked fluorescent markers were used, allowing the identification of the An. gambiae Y through many generations. Y chromosome introgression was confirmed by both genetic tests and DNA sequencing. Anopheles arabiensis males carrying the An. gambiae Y chromosome showed fitness comparable to that of the wild type $A n$. arabiensis males, with no decrease in fertility. This experiment gave an insight into the biology of the $\mathrm{Y}$ chromosome and its role in reproductive isolation. Further, the introgressed strains carrying the An. gambiae Y-linked $a t t P$ and transformation markers are suitable for site-specific integration, mosquito sex separation and could also be used for the application of vector control strategies as discussed for An. gambiae.

\section{Conclusion}

Almost 20 years after the first Anopheles transgenic strain was generated, substantial progress has been made in the field of transgenic technology. This, coupled with the availability of fine molecular tools for genome editing and an increased knowledge of mosquito genetics and biology, has facilitated significant strategic improvements in the research field that aim to vector control. The recent availability of Anopheles strains carrying fluorescent markers or sequences for site-specific integration into the $\mathrm{Y}$ chromosome has revolutionised our ability to separate males from females, which was previously based on inefficient procedures such as differences in the size of pupae. The transgenic sexing strains discussed in this review fulfil the requirements of high-throughput mosquito sex separation without affecting mating competitiveness, which is crucial for making population suppression technologies based on sterile male releases technologies much more powerful. In addition, the Y-linked transgenes are inherited by all-male progeny and no segregation of the sexing markers occur, thus facilitating the maintenance of a pure breeding stock in lab conditions. The potential to introduce a genetic trait specifically onto the Y chromosome allows for the generation of a high throughput sorting method based, for example, on insecticide resistance. Furthermore, a Y-linked male-biased trait could be tailored to benefit novel malaria genetic control programs based on sex-ratio distortion techniques. Finally, the sustainable and effective control of malaria is achievable if the strategies discussed here are advanced through a series of feasibility, safety, efficacy and acceptability studies through combined scientific and social discovery and development efforts. This is the basis for the successful application of transgenic vector control in the field to win the fight against malaria.

\section{Acknowledgments}

This study was benefitted from discussions at International Atomic Energy Agency funded meetings for the Coordinated Research Project "Exploring genetic, molecular, mechanical and behavioural methods of sex separation in mosquitoes." 


\section{Funding}

Publication costs for this study was provided by the International Atomic Energy Agency as part of the Coordinated Research Project "Exploring genetic, molecular, mechanical and behavioural methods of sex separation in mosquitoes."

\section{Availability of data and materials}

Not applicable

\section{About this supplement}

This article has been published as part of Parasites \& Vectors Volume 11 Supplement 2, 2018: Exploring genetic molecular, mechanical and behavioural methods of sex separation in mosquitoes. The full contents of the supplement are available online at https://parasitesandvectors. biomedcentral.com/articles/supplements/volume-11-supplement-2.

\section{Authors' contributions}

BF wrote the manuscript with the support of REH-K, GR, AMH, TN and AC. All authors read and approved the final manuscript.

\section{Ethics approval and consent to participate}

Not applicable

\section{Consent for publication}

Not applicable

\section{Competing interests}

The authors declare that they have no competing interests.

\section{Publisher's Note}

Springer Nature remains neutral with regard to jurisdictional claims in published maps and institutional affiliations.

\section{Published: 24 December 2018}

\section{References}

1. Otto SP. The evolutionary enigma of sex. Am Nat. 2009;174(S1):S1-S14.

2. De Visser JAGM, Ter Maat A, Zonneveld C. Energy budgets and reproductive allocation in the simultaneous hermaphrodite pond snail, Lymnaea stagnalis (L.): a trade-off between male and female function. Am Nat. 1994;144(5): $861-7$.

3. Weinzierl RP, Berthold K, Beukeboom LW, Michiels NK. Reduced male allocation in the parthenogenetic hermaphrodite Dugesia Polychroa. Evolution. 1998:52(1):109-15.

4. Whitton J, Sears CJ, Baack EJ, Otto SP. The Dynamic nature of apomixis in the Angiosperms. Int J Plant Sci. 2008:169(1):169-82.

5. Erickson JW, Quintero JJ. Indirect effects of ploidy suggest $X$ chromosome dose, not the X:A ratio, signals sex in Drosophila. PLoS Biol. 2007:5(12):e332.

6. Baker BS, Wolfner MF. A molecular analysis of doublesex, a bifunctional gene that controls both male and female sexual differentiation in Drosophila melanogaster. Genes Dev. 1988;2(4):477-89.

7. Wilhelm D, Palmer S, Koopman P. Sex determination and gonadal development in mammals. Physiol Rev. 2007:87(1):1-28.

8. WHO, World Malaria Report 2017

9. Baker RH, Sakai RK. Triploids and male determination in the mosquito, Anopheles culicifacies. J Hered. 1979;70(5):345-6.

10. A J, Curtis CF, Davison G. A genetic sexing system in Anopheles gambiae species A. Mosa News. 1976;36:492-8.

11. Krafsur ES. Production of reciprocal translocations in Anopheles gambiae species A. Trans R Soc Trop Med Hyg. 1972;66(1):22-3.

12. Rabbani MG, Kitzmiller JB. Chromosomal translocations in Anopheles albimanus Wiedemann. Mosa News. 1972:32(3):421-32.

13. Rabbani MG, Kitzmiller JB. Studies on $x$-ray induced chromosomal translocations in Anopheles albimanus. II. Laboratory evaluation of sexual competitiveness of translocation males. Am J Trop Med Hyg. 1975;24(6 Pt 1): 1027-30.

14. Krzywinska E, Dennison NJ, Lycett GJ, Krzywinski J. A maleness gene in the malaria mosquito Anopheles gambiae. Science (80- ). 2016;353(6294):67 LP-69.
15. Scali C, Catteruccia F, Li Q, Crisanti A. Identification of sex-specific transcripts of the Anopheles gambiae doublesex gene. J Exp Biol. 2005;208(Pt 19): 3701-9.

16. Criscione F, Qi Y, Saunders R, Hall B, Tu Z. A unique Y gene in the Asian malaria mosquito Anopheles stephensi encodes a small lysine-rich protein and is transcribed at the onset of embryonic development. Insect Mol Biol. 2013:22(4):433-41.

17. Hall $A B$, et al. SEX DETERMINATION. A male-determining factor in the mosquito Aedes aegypti. Science. 2015;348(6240):1268-70.

18. Knipling EF. Possibilities of insect control or eradication through the use of sexually sterile males. J Econ Entomol. 1955:48(4):459-62.

19. Vreysen MJ, et al. Glossina austeni (Diptera: Glossinidae) eradicated on the island of Unguja, Zanzibar, using the sterile insect technique. J Econ Entomol. 2000;93(1):123-35.

20. Dantas L, Pereira R, Silva N, Rodrigues A, Costa R. The SIT control programme against Medfly on Madeira Island. In: Proceedings of the 6th International Symposium on fruit flies of economic importance, Stellenbosch, South Africa, 6-10 May 2002. Irene: Isteg Scientific Publications; 2004. p. 127-30

21. Koyama J, Kakinohana $H$, Miyatake T. Eradication of the melon fly, Bactrocera cucurbitae, in Japan: importance of behavior, ecology, genetics, and evolution. Annu Rev Entomol. 2004;49:331-49.

22. Krafsur ES, Whitten CJ, Novy JE. Screwworm eradication in North and Central America. Parasitol Today. 1987;3(5):131-7.

23. Helinski MEH, Parker AG, Knols BGJ. Radiation biology of mosquitoes. Malar J. 2009;8(2):S6

24. Proverbs MD. Induced sterilization and control of insects. Annu Rev Entomol. 1969:14:81-102

25. A. M. C. Association and E. A. of Mosquito Control Workers, Mosquito News, no. v. 24. American Mosquito Control Association, 1964.

26. Dame DA, Schmidt $\mathrm{CH}$. Uptake of Metepa and its effect on two species of mosquitoes (Anopheles quadrimaculatus, Aedes aegypti) and house flies (Musca domestica). J Econ Entomol. 1964;57(1):77-81

27. Reisen WK, et al. Field competitiveness of Culex tritaeniorhynchus Giles males carrying a complex chromosomal aberration: a second experiment. Ann Entomol Soc Am. 1980;73(4):479-84.

28. Lofgren CS, et al. Release of chemosterilized males for the control of Anopheles albimanus in El Salvador. 3. Field methods and population control. Am J Trop Med Hyg. 1974;23(2):288-97.

29. Bellini R, Medici A, Puggioli A, Balestrino F, Carrieri M. Pilot field trials with Aedes albopictus irradiated sterile males in Italian urban areas. J Med Entomol. 2013;50(2):317-25.

30. Stouthamer R, Breeuwer JA, Hurst GD. Wolbachia pipientis: microbial manipulator of arthropod reproduction. Annu Rev Microbiol. 1999:53:71-102.

31. Zabalou S, Riegler M, Theodorakopoulou M, Stauffer C, Savakis C, Bourtzis K. Wolbachia-induced cytoplasmic incompatibility as a means for insect pest population control. Proc Natl Acad Sci United States Am. 2004:101(42): 15042-5.

32. Atyame CM, Pasteur N, Dumas E, Tortosa P, Tantely ML, Pocquet N, et al. Cytoplasmic Incompatibility as a means of controlling Culex pipiens quinquefasciatus mosquito in the Islands of the South-Western Indian Ocean. PLoS Negl Trop Dis. 2011;5(12):e1440

33. Zhang D, Lees RS, Xi Z, Bourtzis K, Gilles JRL. Combining the sterile insect technique with the incompatible insect technique: III-robust mating competitiveness of irradiated triple Wolbachia-infected Aedes albopictus males under semi-field conditions. PLoS One. 2016;11(3):e0151864.

34. Fisher K. Genetic sexing strains of Mediterranean fruit fly (Diptera: Tephritidae): optimizing high temperature treatment of mass-reared temperature-sensitive lethal strains. J Econ Entomol. 1998;91(6):1406-14.

35. McCombs SD, Lee SG, Saul SH. Translocation-based genetic sexing system to enhance the sterile insect technique against the melon fly (Diptera: Tephritidae). Ann Entomol Soc Am. 1993:86(5):651-4.

36. McCombs SD, Saul SH. Translocation-based genetic sexing system for the oriental fruit fly (Diptera: Tephritidae) based on pupal color dimorphism. Ann Entomol Soc Am. 1995;88(5):695-8.

37. Rössler $Y$. The Genetics of the Mediterranean fruit fly: a 'White Pupae' mutant. Ann Entomol Soc Am. 1979;72(5):583-5.

38. Yamada H, Vreysen MJB, Bourtzis K, Tschirk W, Chadee DD, Gilles JRL. The Anopheles arabiensis genetic sexing strain ANO IPCL1 and its application potential for the sterile insect technique in integrated vector management programmes. Acta Trop. 2015;142:138-44. 
39. Papathanos PA, et al. Sex separation strategies: past experience and new approaches. Malar J. 2009;8(Suppl 2):S5.

40. Ogaugwu CE, Schetelig MF, Wimmer EA. Transgenic sexing system for Ceratitis capitata (Diptera: Tephritidae) based on female-specific embryonic lethality. Insect Biochem Mol Biol. 2013;43(1):1-8.

41. Criscione F, Qi Y, Tu Z. GUY1 confers complete female lethality and is a strong candidate for a male-determining factor in Anopheles stephensi. Elife. 2016;5:e19281.

42. Galizi R, et al. A synthetic sex ratio distortion system for the control of the human malaria mosquito. Nat Commun. 2014;5:3977.

43. Galizi R, et al. A CRISPR-Cas9 sex-ratio distortion system for genetic control. Sci Rep. 2016:6:31139.

44. Hamilton WD. Extraordinary sex ratios. A sex-ratio theory for sex linkage and inbreeding has new implications in cytogenetics and entomology. Science. 1967;156(3774):477-88

45. Schliekelman P, Ellner S, Gould F. Pest control by genetic manipulation of sex ratio. J Econ Entomol. 2005:98(1):18-34.

46. Nolan T, et al. Developing transgenic Anopheles mosquitoes for the sterile insect technique. Genetica. 2011;139(1):33-9.

47. McClintock B. The origin and behavior of mutable loci in maize. Proc Natl Acad Sci. 1950;36(6):344-55.

48. Bellen HJ, O'Kane CJ, Wilson C, Grossniklaus U, Pearson RK, Gehring WJ. Pelement-mediated enhancer detection: a versatile method to study development in Drosophila. Genes Dev. 1989;3(9):1288-300.

49. Spradling AC, Stern DM, Kiss I, Roote J, Laverty T, Rubin GM. Gene disruptions using P transposable elements: an integral component of the Drosophila genome project. Proc Natl Acad Sci U S A. 1995;92(24):10824-30

50. Greenwald I. lin-12, a nematode homeotic gene, is homologous to a set of mammalian proteins that includes epidermal growth factor. Cell. 1985;43(3 Pt 2):583-90.

51. Moerman DG, Benian GM, Waterston RH. Molecular cloning of the muscle gene unc-22 in Caenorhabditis elegans by Tc1 transposon tagging. Proc Natl Acad Sci U S A. 1986;83(8):2579-83.

52. Osborne BI, Baker B. Movers and shakers: maize transposons as tools for analyzing other plant genomes. Curr Opin Cell Biol. 1995;7(3):406-13.

53. Wilson MH, Coates CJ, George ALJ. PiggyBac transposon-mediated gene transfer in human cells. Mol Ther. 2007;15(1):139-45.

54. Rubin GM, Spradling AC. Genetic transformation of Drosophila with transposable element vectors. Science. 1982;218(4570):348-53.

55. SanMiguel $P$, et al. Nested retrotransposons in the intergenic regions of the maize genome. Science. 1996;274(5288):765-8.

56. Catteruccia F, et al. Stable germline transformation of the malaria mosquito Anopheles stephensi. Nature. 2000;405(6789):959-62.

57. Grossman GL, Rafferty CS, Clayton JR, Stevens TK, Mukabayire O, Benedict MQ. Germline transformation of the malaria vector, Anopheles gambiae, with the piggyBac transposable element. Insect Mol Biol. 2001;10(6):597-604.

58. Perera OP, Harrell II RA, Handler AM. Germ-line transformation of the South American malaria vector, Anopheles albimanus, with a piggyBac/EGFP transposon vector is routine and highly efficient. Insect Mol Biol. 2002;11(4): 291-7.

59. Hall $A B$, et al. Radical remodeling of the $Y$ chromosome in a recent radiation of malaria mosquitoes. Proc Natl Acad Sci. 2016;113(15):201525164.

60. Bernardini F, et al. Site-specific genetic engineering of the Anopheles gambiae Y chromosome. Proc Natl Acad Sci. 2014;111(21):201404996.

61. Catteruccia F, Benton JP, Crisanti A. An Anopheles transgenic sexing strain for vector control. Nat Biotechnol. 2005;23(11):1414-7.

62. Bernardini $F$, et al. Cross-species $Y$ chromosome function between malaria vectors of the Anopheles gambiae species complex. Genetics. 2017;207:729

63. Marois E, Scali C, Soichot J, Kappler C, Levashina EA, Catteruccia F. Highthroughput sorting of mosquito larvae for laboratory studies and for future vector control interventions. Malar J. 2012;11(1):302.

64. Thorpe HM, Smith MCM. In vitro site-specific integration of bacteriophage DNA catalyzed by a recombinase of the resolvase/invertase family. Proc Natl Acad Sci U S A. 1998;95(10):5505-10.

65. Meredith $\mathbf{J M}$, et al. Site-specific integration and expression of an antimalarial gene in transgenic Anopheles gambiae significantly reduces Plasmodium infections. PLoS One. 2011;6(1):e14587.

66. Franz G. Genetic sexing strains in Mediterranean fruit fly, an example for other species amenable to large-scale rearing for the sterile insect technique. In: Dyck VA, Hendrichs J, Robinson AS, editors. Sterile Insect
Technique: Principles and Practice in Area-Wide Integrated Pest Management. Dordrecht: Springer Netherlands; 2005. p. 427-51.

67. Curtis CF. Genetic sex separation in Anopheles arabiensis and the production of sterile hybrids. Bull World Health Organ. 1978;56(3):453-4.

68. C. Curtis Genetic sexing techniques based on translocations of insecticide resistance to the $Y$ chromosome [genetic control of pests] Bulletin SROP (OIBC), vol. v. 2. 1979.

69. Kaiser PE, Seawright JA, Dame DA, Joslyn DJ. Development of a genetic sexing system for Anopheles albimanus. J Econ Entomol. 1978;71(5):766-71.

70. Yamada H, Benedict MQ, Malcolm CA, Oliva CF, Soliban SM, Gilles JRL. Genetic sex separation of the malaria vector, Anopheles arabiensis, by exposing eggs to dieldrin. Malar J. 2012;11:208.

71. Jinek M, Chylinski K, Fonfara I, Hauer M, Doudna JA, Charpentier E. A programmable dual-RNA-guided DNA endonuclease in adaptive bacterial immunity. Science. 2012:337(6096):816-21.

72. Gantz VM, et al. Highly efficient Cas9-mediated gene drive for population modification of the malaria vector mosquito Anopheles stephensi. Proc Natl Acad Sci U S A. 2015;112(49):E6736-43.

73. Hammond A, et al. A CRISPR-Cas9 gene drive system targeting female reproduction in the malaria mosquito vector Anopheles gambiae. Nat Biotechnol. 2016;34(1):78-83.

74. Haghighat-Khah RE, et al. Site-specific cassette exchange systems in the Aedes aegypti mosquito and the Plutella xylostella moth. PLoS One. 2015; 10(4):e0121097.

75. Charlesworth B. Evoluton of sex chromosomes. Science (80- ). 1991;251: 1030-3.

76. Rice WR. Evolution of the $Y$ sex in animals: $Y$ chromosomes evolve through the degeneration of autosomes. Bioscience. 1996;46(5):331-43.

77. Charlesworth B, Charlesworth D. The degeneration of $Y$ chromosomes. Philos Trans R Soc B-Biological Sci. 2000;355(1403):1563-72.

78. Bachtrog D. Y chromosome evolution: emerging insights into processes of Y chromosome degeneration. Nat Rev Genet. 2013;14(2):113-24.

79. Schneider P, Takken W, McCall PJ. Interspecific competition between sibling species larvae of Anopheles arabiensis and An. gambiae. Med Vet Entomol. 2000;14(2):165-70

80. Neafsey D, Waterhouse RM, Abai MR, Aganezov SS, Alekseyev MA, Allen JE, et al. Highly evolvable malaria vectors: the genomes of 16 Anopheles mosquitoes. Science (80- ). 2015;347(6217):1258522.

\section{Ready to submit your research? Choose BMC and benefit from:}

- fast, convenient online submission

- thorough peer review by experienced researchers in your field

- rapid publication on acceptance

- support for research data, including large and complex data types

- gold Open Access which fosters wider collaboration and increased citations

- maximum visibility for your research: over $100 \mathrm{M}$ website views per year

At $\mathrm{BMC}$, research is always in progress.

Learn more biomedcentral.com/submission 